\title{
Bladder pain syndrome from urogynecological point of view: a narrative
}

\author{
Salah E. Shebl' ${ }^{1}$ Ayman S. Dawood ${ }^{2 *}$, Abdel-Ghaffar S. Dawood ${ }^{2}$
}

${ }^{1}$ Department of Urology, Al Azhar University, Cairo, Egypt
${ }^{2}$ Department of Obstetrics and Gynecology, Tanta University, Tanta, Egypt

Received: 26 December 2016

Accepted: 23 February 2017

\section{*Correspondence:}

Dr. Ayman S. Dawood,

E-mail: ayman.dawood@med.tanta.edu.eg

Copyright: (c) the author(s), publisher and licensee Medip Academy. This is an open-access article distributed under the terms of the Creative Commons Attribution Non-Commercial License, which permits unrestricted non-commercial use, distribution, and reproduction in any medium, provided the original work is properly cited.

\begin{abstract}
Hypothesis: bladder pain syndrome (BPS) presents by both urological and gynecological symptoms. The gynecological symptoms include chronic persistent pelvic pain, dyspareunia and decreased libido on the other hand urological symptoms include wide variety of symptoms as bladder discomfort, dysuria, frequency, and urgency. Although, the definition and diagnostic criteria for the condition was established, the pathogenesis, etiology and histologic findings of BPS are still not fully understood and diagnosis is made by exclusion. Review of published data including both gynecological and urological articles focusing on the updates in the diagnostic tests, pathology and the recent therapies. The searched words were bladder pain syndrome, interstitial cystitis and painful bladder. The free full text article found after our search were 454 articles after exclusion of deficient article not covering the whole subject, 91 articles were enough for this work to be completed. From this review, Bladder pain syndrome is still unclear disease lacking curative therapy up till now and patients are still suffering and the mixed complex symptoms put further challenge on both urologists and gynecologists to solve the problem.
\end{abstract}

Keywords: Bladder pain syndrome, Diagnosis, Management, Pathogenesis

\section{INTRODUCTION}

In 2002, the International Incontinence Society defined BPS by "the complaint of suprapubic pain related to bladder filling, accompanied by other symptoms such as increased daytime and night time frequency without evidence of proven urinary infection or other obvious pathology". 1

European Society for the study of BPS (ESSIC) proposed the change of name of the condition from Interstitial Cystitis to BPS. BPS is diagnosed by the presence of chronic pelvic pain lasting more than 6 months, pressure/discomfort perceived to be related to the urinary bladder, and one or more urinary symptoms such as urinary urgency or frequency. ${ }^{2}$ The American Urological Association defined IC/BPS as follows "An unpleasant sensation (pain, pressure, or discomfort) perceived to be related to the urinary bladder, and associated with lower urinary tract symptoms of more than six weeks duration in the absence of infection or other identifiable cause". ${ }^{3}$

Painful bladder syndrome (PBS) is multi-symptom syndrome presenting by chronic pelvic pains, dysuria, frequency and sometimes urgency.

\section{CLASSIFICATIONS}

BPS is classified into 3 types: 1, 2, and 3 according to cystoscopic findings;

- Type 1: Normal cystoscopic findings.

- Type 2: There are glomerulation

- Type 3: Hunner's lesion is present 
Each type is composed 3 capital letters A, B and C according to histologic findings;

- A: Normal

- B: Inconclusive

- C: Positive

Each type has 2 symbols for example; BPS type 1A means patients with normal finding at cystoscopy and normal histology on biopsy. ${ }^{2}$

\section{EPIDEMIOLOGY}

There is a great difficulty to determine the actual incidence of this condition due to absence of accurate diagnostic tests, dispute between urology and gynecology for eligibility for treatment plus deficient registration. ${ }^{4}$

Berry et al used the questionnaire to determine prevalence of BPS among adult females in the US. This study yielded prevalence estimates of from $2.7 \%$ to $6.53 \%$ (approximately 3.3 to 7.9 million US women age 18 or older). Only $9.7 \%$ of women who met the definitions reported having been given an BPS diagnosis. ${ }^{5}$

In Europe the prevalence was reported to be 18 / 100,000 women, whereas in Japan only 3-4 cases per 100,000 women were reported. The differences between these countries and the United States are marked and are due to differences in diagnostic criteria and clinical criteria. ${ }^{6}$

As regard age incidence, the median age at presentation is 40 years. However, BPS may occur in children. ${ }^{7}$ BPS is more in white races $(94 \%)$ and more in females approximately $90 \%$. BPS is slightly more common in Jewish women. ${ }^{8}$

\section{PATHOLOGY}

\section{Histologic picture}

The positive histologic evidence of BPS includes inflammatory infiltrate and/or granulation tissue and/or detrusor mastocytosis and/or intra-fascicular fibrosis. ${ }^{9}$

The inflammatory infiltrates are usually superficial, and restricted to the lamina propria. The lamina propria has edema, with hemorrhage and congested venules in the stroma.

Hemorrhage of the lamina propria is more common in patients with ulcers than those without ulcers. Evidence of fibrosis in detrusor muscle is present in $10 \%$ of patients, and is pathognomonic to patients with ulcer. ${ }^{9}$

Tamm-Horsfall protein is deposited in the epithelium and submucosa in patients with BPS, indicating a barrier defect in this disease. ${ }^{9}$
Recently, pathologic investigators suggested that patients with BPS had prominent plasma cell infiltration and fibrosis in the affected bladder tissue, raising the suspicion that BPS could be part of a systemic IgG4related disease. Their study showed $60 \%$ IgG4 positivity in both serum IgG4 level and IgG4-to-IgG ratio. They proposed that a BPS may be due to IgG4-related disease and this potentially provides a clear idea about the pathogenesis of IC. ${ }^{10}$

\section{Pathogenesis}

BPS pathophysiology may be explained by:

- Neural hypersensitivity

- Immune or inflammatory abnormal signals

- Disruption of the proteoglycan/glycosaminoglycan (GAG) layer. ${ }^{11}$

Neurogenic inflammation: Many mediators of neurogenic inflammation, such as substance $\mathrm{P}$, and neurokinin $\mathrm{A}$ are increased in BPS. Additionally, up-regulation of neural afferent pathways has been shown in BPS, as well as a central hyperresponsiveness and hypersensitivity of neural receptors to these mediators. ${ }^{12-14}$

Infectious etiology this pathogenic theory is supported by increased mast cells and increased mediators of infection in the epithelium and bladder wall. Antibiotic treatment was not effective and infection may be just a trigger for the BPS. ${ }^{15}$

Disruption of glycosaminoglycan layer leads to a leak of toxic substances, allergens or bacteria through bladder epithelium to the deep layers of bladder wall and cause an inflammatory process. It is still unclear whether the increased permeability of bladder epithelium in patients with BPS is a step in the pathophysiology or a consequence of an inflammatory cascade. Hence, restoration of integrity of bladder epithelial layer is the cornerstone in treatment if BPS. ${ }^{16}$

\section{Cystoscopic picture}

BPS has 2 distinct subgroups based on findings at cystoscopy and bladder overdistention. These categories are the ulcerative (ie, classic) and non-ulcerative (ie, Messing-Stamey) types. Evidence showing progression of ulcerative to nonulcerative disease, or vice versa, is lacking. ${ }^{16}$

\section{Ulcerative}

The surface epithelium shows diffuse redness associated with 1 or more ulcerative patches surrounded by congested mucosa (ie, Hunner ulcer). These ulcers are seen on the dome or lateral walls of the bladder. These ulcers may become apparent only after overdistention which leads to fissures and cracks in the bladder epithelium. 
The ulcerative type is less common and present in less than $10 \%$ of cases. This type is more resistant to therapy. Biopsy findings show that the ulcerative lesion may be transmural and associated with marked inflammatory changesand fibrosis. This classic type leads to progressive decrease in bladder capacity over time. ${ }^{17}$

\section{Non-ulcerative}

This type is characterized absent cystoscopic findings noted in the ulcerative type. After overdistention, glomerulations are discreet, small mucosal tears and hemorrhages; appear on the dome and lateral walls of the bladder. Bladder biopsy findings are often unremarkable when compared to those found in ulcerative type. ${ }^{18}$

\section{Biomarkers of BPS}

Bladder nitric oxide is an accurate marker for Hunner lesions, but these are not present in all patients, and the test requires specific equipment, which has limites its clinical use. ${ }^{19}$

Cytokines and chemokines, specifically CXCL-10, differentiate patients with and without Hunner lesions. Other studies of ulcerative BPS showed that numerous other cytokines and chemokines are detected as well, impeding their clinical significance to identify patients. ${ }^{20,21}$

Antiproliferative factor (APF). This small 8-amino-acid peptide is associated with suppression of cell growth, increases in transcellular permeability, and decrease in proteins of intercellular junction complexes. It is synthesized and secreted from bladder epithelial cells in patients with BPS and may play a key role in pathophysiology. It is considered the most promising urinary biomarker in BPS. ${ }^{22}$

Gene analysis of urothelial proinflammatory markers has been actively tried recently including CXCR3 cytokine, TNFSF14, COX-2 and others. ${ }^{23}$

\section{ETIOLOGY}

\section{Risk factors}

The only definitive risk factor for PBS is female gender, the female-to-male ratio is generally estimated to be $9: 1$ Other risk factors that have been proposed include heredity and previous urinary tract infection. ${ }^{24}$

Heredity might play a role in the pathogenesis of BPS. In 2004 study found that prevalence of BPS in females who have a first-degree relative with confirmed BPS is 1,431 per 100,000.5 In comparison, the prevalence rate in the general population is estimated to be about 60 per 100,000. The 2004 study also found that the prevalence of BPS in first-degree female relatives of patients with
PBS confirmed by NIDDK diagnostic criteria was 17 times higher than the rate in the general population. ${ }^{25,26}$

\section{Causes}

The etiology of interstitial cystitis remains unknown and is likely multifactorial. Proposed etiologies include the following:

- Pathogenic role of mast cells in the detrusor and/or mucosal layers of the bladder. ${ }^{27}$

- Deficiency in the glycosaminoglycan layer on the luminal surface of the bladder, resulting in increased permeability of the underlying submucosal tissues to toxic substances in the urine. ${ }^{28}$

- Infection with a poorly characterized agent (eg, a slow-growing virus or extremely fastidious bacterium). ${ }^{15}$

- Neurogenic hypersensitivity or inflammation mediated locally at the bladder or spinal cord level. ${ }^{12-}$ 14

- Manifestation of pelvic floor muscle dysfunction or dysfunctional voiding. ${ }^{29}$

- Autoimmune disorder. BPS is associated by other autoimmune conditions like Sjögren syndrome and fibromyalgia syndrome showing antibodies against urothelium, smooth muscle. ${ }^{30}$

- Psychiatric conditions associated with interstitial cystitis include anxiety disorder, depression, and adjustment reactions. ${ }^{31}$

\section{CLINICAL PICTURE}

\section{Symptoms}

\section{Pain}

Is the hallmark symptom of BPS. Typical BPS patients complain of suprapubic pain, pressure or discomfort related to bladder filling. Pain may be felt throughout the pelvis in the urethra, vulva, vagina, and rectum. Pain may be felt in extragenital sites such as the lower abdomen and back. ${ }^{32-34}$ Warren and colleagues found that by using "pelvic pain" as the key descriptor that $100 \%$ of his population fit the case definition. ${ }^{35-37}$ Pain may be worsened with specific foods, drinks and/or worsened with bladder filling and is improved with urination. ${ }^{38}$

\section{Urinary manifestations}

Which accompany BPS and are not specific such as urgency and frequency because these symptoms may indicate other disorders, not exclusively indicate the presence of BPS. Frequency is a universal symptom being present in $92 \%$ of urinary patients and does not distinguish BPS from other lower urinary tract disorders. Urinary urgency is also extremely common being present in $84 \%$ of urinary patients. ${ }^{39}$ Urgency is a characteristic symptom of overactive bladder, but a qualitative 
difference exists between patients with the 2 conditions. $^{40,41}$ Patients with BPS void to avoid or to relieve pain; while $\mathrm{OAB}$ patients, void to avoid incontinence. Symptoms of urinary urgency and frequency may precede symptoms of pain. ${ }^{42}$

\section{- Gynecological manifestations}

\section{Chronic pelvic pain ${ }^{43}$}

Pain is the main symptom of BPS, and most patients do not have a specific site for pain or fail to localize it into the bladder. Pain usually is complex and felt beyond the bladder in (vagina, lower abdomen, lower back, pelvis, and buttocks). Those patients soak medical advice at multiple clinics of urologists and gynecologists with many unsuccessful management trials.

Sexual dysfunction including orgasmic difficulties, sexual pain disorder, inadequate lubrication, hypoactive desire. These patients consequently had poor quality of life and lowerd self-esteem. ${ }^{44}$

\section{Signs}

Careful history and physical examination are crucial for diagnosis of those patients with BPS and multidisciplinary approach has to be followed in all cases including both gynecologists and urologists for making a real improvement in the patient's symptoms. ${ }^{45}$

Abdominal, pelvic, and directed neurologic examinations should be performed in all patients with voiding dysfunction. Nevertheless, the findings from these examinations are often unrevealing in patients with interstitial cystitis. Women with interstitial cystitis may express some discomfort with palpation over the urethra and bladder base. A correlation has been noted between urethral tenderness and the finding of a Hunner ulcer on cystoscopic examination. ${ }^{45}$

Pain upon urethral palpation in the presence of an anterior vaginal wall mass may suggest urethral diverticulum, whereas cervical motion tenderness may suggest pelvic inflammatory disease. On examination with a speculum, any of the following findings suggest a diagnosis other than interstitial cystitis: ${ }^{45}$

- Prolapse

- Masses

- Evidence of vaginitis, herpes, vestibular adenitis, vulvovestibulitis, vulvodynia, or other pathology.

Palpation for a full bladder and bimanual examination evaluating for adnexal masses should be part of the complete examination. Rectal examination should always be performed to evaluate for masses, tenderness, and assessment of rectal and pelvic floor muscle tone. ${ }^{45}$
Neurologic examination findings are usually unremarkable, but abnormalities of motor function, sensation, or reflexes may indicate spinal cord or nerve root dysfunction and should prompt further evaluation for other diagnoses. ${ }^{45}$

\section{LITERATURE REVIEW}

Review of literature was commenced in the period from 2000 to 2016 on MEDLINE, PUBMED, CROSSREF, RESEARCH GATE, CLINICAL KEY and GOOGLE SCHOLAR especially clinical trials and systematic reviews to provide a sufficient review on this condition including both gynecological and urological aspects focusing on the updates in the diagnostic tests, pathology and the recent therapies. The search words were bladder pain syndrome, interstitial cystitis and painful bladder. The free full text article found after our search were 454 articles after exclusion of deficient non-relevant articles, 91 articles were enough for this work to be completed.

\section{DIAGNOSIS}

The basic assessment should include a careful history, physical examination, and laboratory examination to rule in symptoms that characterize BPS and rule out other confusable disorders. Baseline voiding symptoms and pain levels should be obtained in order to measure subsequent treatment effects. The diagnosis of IC is based on the presence of pain related to the bladder, usually accompanied by frequency and urgency absence of other diseases that could cause the symptoms. The physical examination usually includes a complete pelvic examination with a brief rectal exam. Often, patients with BPS have tenderness in the lower abdomen, hips, and buttocks. Women often have tenderness in the vagina and around the bladder. ${ }^{47}$

- Urine test: Urine analysis, culture and sensitivity to rule out presence of infection

- Ultrasound scanning to assess post-void residual urine volume

- The $\mathrm{KCl}$ test, also known as the potassium sensitivity test, is no longer recommended. The test uses a mild potassium solution to evaluate the integrity of the bladder wall. Though the latter is not specific for BPS, it has been determined to be helpful in predicting the use of compounds, such as pentosan polysulphate, which are designed to help repair the GAG layer

- Uroflowmetry and Urodynamic study should be considered as an aid to diagnosis only for complex presentations; these tests are not necessary for making the diagnosis in uncomplicated presentations.

- Cystoscopy with hydrodistention to visualize the bladder wall after stretching the bladder was not specific for BPS and that the test, itself, can contribute to the development of small glomerulations (petechial hemorrhages) often found 
in BPS. Thus, a diagnosis of BPS is one of exclusion, as well as a review of clinical symptoms

- Biopsy. During cystoscopy under anesthesia. ${ }^{46,47}$

\section{MANAGEMENT}

Management of BPS/IC requires a multimodal approach. The AUA and the European Urological Association have produced management guidelines for BPS/IC, both of which were updated in 2014.

Conservative management is considered the first line therapy for IC is in form of education, behavioral modification, and stress control. Patient education as to the normal function of the bladder as well as what behaviors may lead to increased bladder pain is integral to symptom control. Strategies may include: altering diet to avoid bladder irritants such as caffeine and spicy foods, altering urine volume to control concentration. ${ }^{48,49}$

According to guidelines of European Association of Urology, the available treatment options for patients with BPS included the oral pentosan polysulfate sodium and intravesical Dimethyl sulfoxide (DMSO) which are both FDA-approved drugs Botulinum neurotoxins (BoNTs), which are produced by the bacterium Clostridium botulinum, have been widely applied medically since the first report of BoNTs for ocular strabismus in 1980 50,51

\section{Medical (oral pharmacological therapy)}

- Pain control: For those patients with failed conservative management

- Amitriptyline: A tricyclic antidepressant that has been for neuropathic pain

- Hydroxyzine/cimetidine: An H1-receptor agonist and an H2-receptor antagonist, respectively. These drugs acting by preventing histamine release and mast cell degranulation (one of the mechanisms that has been suggested in the pathophysiology of IC)..$^{52}$

- Pentosan polysulfate (PPS): The mechanism of action is to repair the glycosaminoglycan (GAG) layer of the bladder urothelium and reducing its permeability. ${ }^{53}$

- Cyclosporine A (CyA): An oral drug, used for immunomodulation in transplant recipients and certain autoimmune disorders. CyA th mechanism of action is to inhibit calcineurin, which is necessary for the activation of $\mathrm{T}$ cells so it may has abenefit in patients with bladder inflammation caused by IC. ${ }^{54}$

\section{Intra-vesical therapies}

\section{Dimethyl sulfoxide (DMSO)}

It is an organosulfur non-toxic solvent. DMSO is used in the treatment of BPS since the 1970's when it was approved by the US Food and Drug Administration (FDA) for intra-vesical use. It acts through several mechanisms: reducing inflammation, acting as an analgesic and facilitating detrusor relaxation. It can be given as a single-agent instillation at a $50 \%$ concentration or, more commonly, as part of a 'cocktail' with methylprednisolone or hydrocortisone, alkalised lidocaine and heparin sulfate. Instillation regimens differ but a common treatment program involves twice weekly instillations for 4 weeks and then weekly instillations for a further 4 weeks, for a total of 12 instillations. Repeat full, partial (four weekly instillations) or maintenance (monthly) treatment courses for persistent or recurrent symptoms are possible. ${ }^{55}$

\section{Heparin and lidocaine}

It helps in regeneration of the urothelial GAG layer, inhibits fibroblast proliferation and promotes angiogenesis and smooth muscle cell proliferation. Lidocaine is a topical anaesthetic and is used as a singleagent instillation or, more commonly, in combination with heparin. It is administrated usually in combination with an alkalising agent (sodium bicarbonate) to avoid ionisation within urine and to better penetrate the urothelium 56

\section{Pentosan polysulfate sodium (PPS)}

Orally approved for BPS by the FDA. It acts by restoring the GAG layer, inhibit histamine release by mast cells and reduces the intracellular calcium ion levels in the bladde. $^{57}$

\section{Sodium hyaluronate $(H A)$}

It is having a role in enhancement of connective tissue healing and inhibition of leukocyte migration and aggregation may contribute to its action in BPS/IC patients. It is commercially available as Cystistat (Teva UK Limited); it comes as a $40 \mathrm{mg}$ dose in a $50 \mathrm{~mL}$ solution. The mostly used regimen consisting of four weekly instillations followed by monthly therapy until symptoms have resolved. ${ }^{57}$

\section{Chondroitin sulfate (CS)}

CS is a component of the GAG layer and has been shown to be deficient in patients with BPS/IC. As well as its role in the GAG layer, $\mathrm{CS}$ has been shown to inhibit the recruitment of inflammatory cells to the deep layers of the bladder wall. ${ }^{58}$

\section{Sodium hyaluronate and chondroitin sulfate (HA-CS)}

It reduces the production of pro-inflammatory cytokines, reduce urothelial permeability, and facilitate the repair of the protective GAG layer. ${ }^{58}$ 


\section{Oxychlorosene sodium (OS)}

An antibacterial agent used in general surgery, particularly in the irrigation of wounds. The definite mechanism of action is unclear but it may act by desensitizing or degranulation bladder nociceptive nerve endings..$^{59}$

\section{Botulinum toxin A}

Powerful neurotoxin acts by inhibiting the release of acetylcholine and other neurotransmitters from both afferent and efferent nerve terminals as well as ATP from the urothelium. Chronic inflammation and apoptotic signalling molecules is significantly reduced following intra-vesical botulinum toxin A injections, but only after repeated injections. The recently amended AUA guidelines for the treatment of BPS/IC have moved botulinum toxin $\mathrm{A}$ from 5 th to first 4 th line treatment. ${ }^{59}$

\section{Other intra-vesical therapies}

- Capsaicin and resiniferatoxin are C-fiber afferent neurotoxins. It has theoretical ability to alleviate bladder symptoms by desensitising bladder afferents. Recently not recommended for intravesical use in BPS/IC patients. ${ }^{60}$

- Bacillus Calmette-Guerin (BCG) is an immunomodulator used for the intravesical treatment of noninvasive bladder cancer. Currently is not recommended for use in BPS/IC patients due to adverse serious effects. ${ }^{61}$

- Liposomes are phospholipid vesicles believed that they may be able to restore the GAG layer by intravesical liposomal instillations. ${ }^{62}$

\section{Surgical therapy}

\section{Cystoscopy with hydrodistention/Fulguration of lesions}

Hydrodistension under anesthesia with low-pressure Cystoscopy (60 to $80 \mathrm{cmH}_{2} \mathrm{O}$ ), short duration (less than 10 minutes). This has several values by allowing inspection of the bladder for glomerulations and Hunner's ulcers or any other bladder lesions. It also allows for staging by measuring anatomical capacity (rather than just functional capacity). ${ }^{63}$

The hydrodistention allows for the break down and subsequent reconstruction of damaged nerve pathways, even in the absence of lesions also allows for the examination of the bladder for Hunner's lesions. These lesions present as a reddened mucosal area with small vessels radiating towards a central scar, and have increased likelihood of bleeding, especially during hydrodistention. Although relatively rare (occurring in only $5-10 \%$ of patients), identification of Hunner's lesions is important because treatment appears to constitute one of the few IC therapies that results in significant prolonged improvement with only a single exposure to the procedure. Fulguration or sclerosing of Hunner's lesions has been demonstrated multiple times to result in complete or almost complete resolution of pain symptoms for durations of over a. ${ }^{64}$

\section{Intra-detrusor botulinum toxin A (BTX-A)}

For the treatment of BPS, current AUA guidelines list BTX-A as an option with grade C evidence. This option is based upon one randomized control trial and nine observational studies that demonstrated highly variable amounts of short and long efficacy rates (from $20 \%$ to $86 \%$ at 3 months). ${ }^{59}$

Bladder augmentation, urinary diversion, and resection of ulcers, and cystectomy

Rarely used, and is considered a treatment of last choice for severe refractory cases of interstitial cystitis. ${ }^{65}$

\section{Physical medicine}

For strengthening of pelvic floor musculature, some physical exercises have been used to treat BPS.

\section{Neuromodulation}

Neuromodulation through sacral nerve stimulation (SNS) involves an initial test phase with insertion of a test lead tunneled under the skin transmitted onto the nerve roots exiting the S3 foramen. The pelvic and pudendal nerves are stimulated by an external stimulator that is later exchanged for a permanent implant if successful. This technique has been shown to be effective in several disease states including fecal incontinence, urinary urgency, urge incontinence and urinary retention and has an FDA indication for these symptoms. ${ }^{47}$

\section{Bladder training}

Bladder training is a self-control technique suppressing urge to urinate, which has been listed as the first-line treatment in AUA guideline. ${ }^{47}$

\section{Biofeedback}

Is a technique enhancing the effect of pelvic floor muscle exercises, has been used to treat various urological diseases, such as stress urinary incontinence and overactive bladder. However, the role of biofeedback in managing BPS symptoms is debatable. It has been revealed that pelvic floor muscle over-activity is an important factor contributing to BPS symptoms. Biofeedback may relieve BPS symptoms by lowering resting pelvic floor muscular tone. ${ }^{67}$

Yoga

Yoga is a mixture of physical and mental exercise that originated in ancient India. A systematic review showed 
that yoga can effectively relieve chronic pain in a variety of disorders. ${ }^{67}$

\section{Massage}

Massage refers to a series of actions on the body with appropriate pressure to obtain muscular relaxation. ${ }^{55}$

\section{Herbal and alternative medicine}

Dietary modifications have been recommended as firstline treatment of BPS in American Urological Association (AUA) guideline. ${ }^{68}$

\section{Nutraceuticals}

Nutraceuticals are the products isolated from food, plant and herb, which can improve health and prevent chronic diseases. Some nutraceuticals have been used to relieve BPS symptoms.

L-Arginine: L-arginine is a semi-essential amino acid, which can be converted to nitric oxide (NO) by NO synthase related enzymes. NO is an important regulator for multiple physiological functions of humans. A systematic review revealed that NO pathway plays a major role in regulating lower urinary tract function. Not only can NO modulate bladder afferent neurons activity, but relax the urethral smooth muscle cells. ${ }^{69}$

\section{Herbal therapy}

Herbal therapy has been used for many urological diseases over the past decades and the effectiveness has been confirmed. However, the use of herbal medicine for BPS remains controversial because the efficacy is variable. $^{70}$

\section{Energy therapies ex. acupuncture}

An effective therapy, has been accepted by urologists over the past decades. ${ }^{71}$

Funding: No funding sources

Conflict of interest: None declared

Ethical approval: Not required

\section{REFERENCES}

1. Abrams P, Cardozo L, Fall M, Griffiths D, Rosier P, Ulmsten U, et al. The standardisation of terminology of lower urinary tract function: report from the Standardisation Sub-committee of the International Continence Society. Neurourol Urodyn. 2002;21:167-78.

2. van De Merwe JP, Nordling J, Bouchelouche P, Bouchelouche K, Cervigni M, Daha LK, et al. Diagnostic criteria, classification, and nomenclature for painful bladder syndrome/interstitial cystitis: an ESSIC proposal. Eur Urol. 2008;53:60-7.

3. Hanno P, Dmochowski R. Status of international consensus on interstitial cystitis/bladder pain syndrome/painful bladder syndrome: 2008 snapshot. Neurourol Urodyn. 2009;28:274-86.

4. Clemens JQ, Joyce GF, Wise M. Interstitial cystitis and painful bladder syndrome. In: Urologic Diseases in America. Edited by M. S. Litwin and C. S. Saigal. Washington, DC: US Department of Healt and Human Services, Public Health Service, National Institutes of Health, National Institute of Diabetes and Digestive and Kidney Disease; 2007:125-154.

5. Berry SH. Prevalence of symptoms of bladder pain syndrome/interstitial cystitis among adult females in the United States. J Urol. 2011;186:540.

6. Berry SH, Bogart LM, Pham C, Liu K, Nyberg L, Stoto M. Development, validation and testing of an epidemiological case definition of interstitial cystitis/painful bladder syndrome. J Urol. 2010;183(5):1848-52.

7. Yoost JL, Hertweck SP, Loveless M. Diagnosis and treatment of interstitial cystitis in adolescents. J Pediatr Adolesc Gynecol. 2012;25(3):162-71.

8. Konkle KS, Berry SH, Elliott MN, Hilton L, Suttorp MJ, Clauw DJ, et al. Comparison of an interstitial cystitis/bladder pain syndrome clinical cohort with symptomatic community women from the RAND Interstitial Cystitis Epidemiology study. J Urol. 2012;187(2):508-12.

9. Lopez-Beltran A, Montironi R, Cheng L. Pathology of the urinary bladder. Cambridge University Press; 2016:48-66.

10. Crumley S, Ge Y, Zhou H, Shen SS, Ro JY. Interstitial cystitis: another IgG4-related inflammatory disease? Ann Diagn Pathol. 2013;17:403-7.

11. Dyer AJ, Twiss CO. Painful bladder syndrome: an update and review of current management strategies. Curr Urol Rep. 2014;15(2):384.

12. Mukerji G, Yiangou Y, Grogono J, Underwood J, Agarwal SK, Khullar V. Localization of M2 and M3 muscarinic receptors in human bladder disorders and their clinical correlations. J Urol. 2006;176(1):36773.

13. Nazif O, Teichman JM, Gebhart GF. Neural upregulation in interstitial cystitis. Urology. 2007;69(4 Suppl):24-33.

14. Twiss C, Kilpatrick L, Craske M, Buffington CA, Ornitz E, Rodríguez LV. Increased startle responses in interstitial cystitis: evidence for central hyperresponsiveness to visceral related threat. J Urol. 2009;181(5):2127-33.

15. Keay SK, Warren JW. Is interstitial cystitis an infectious disease? Int $\mathrm{J}$ Antimicro Agents. 2002;19(6):480-3.

16. Ford AP, Gever JR, Nunn PA, Zhong Y, Cefalu JS, Dillon MP, et al. Purinoceptors as therapeutic targets for lower urinary tract dysfunction. Br J Pharmacol. 2006;147(2):S132-43. 
17. Peters KM, Jaeger C, Killinger KA, Rosenberg B, Boura JA. Cystectomy for ulcerative interstitial cystitis: sequelae and patients' perceptions of improvement. Urol. 2013;82(4):829-33.

18. Hanno PM, Erickson D, Moldwin R, Faraday MM. American Urological, Association. Diagnosis and treatment of interstitial cystitis/bladder pain syndrome: AUA guideline amendment. J Urol. 2015;193(5):1545-53.

19. Hosseini A, Ehrén I, Wiklund NP. Nitric oxide as an objective marker for evaluation of treatment response in patients with classic interstitial cystitis. J Urol. 2004;172(6 Pt 1):2261-5.

20. Tyagi P, Killinger K, Tyagi V, Nirmal J, Chancellor $\mathrm{M}$, Peters KM. Urinary chemokines as noninvasive predictors of ulcerative interstitial cystitis. J Urol. 2012;187(6):2243-8.

21. Blalock EM, Korrect GS, Stromberg AJ, Erickson DR. Gene expression analysis of urine sediment: evaluation for potential noninvasive markers of interstitial cystitis/bladder pain syndrome. J Urol. 2012;187(2):725-32.

22. Kim J, Freeman MR. Antiproliferative factor signaling and interstitial cystitis/painful bladder syndrome. Int Neurourol J. 2011;15(4):184-91.

23. Kim HJ. Update on the pathology and diagnosis of interstitial cystitis/bladder pain syndrome: a review. Int Neurourol J. 2016;20(1):13-7.

24. Jhang JF, Kuo HC. Pathomechanism of Interstitial Cystitis/Bladder Pain Syndrome and Mapping the Heterogeneity of Disease. Int Neurourol J. 2016;20(2):95-104.

25. Warren JW, Jackson TL, Langenberg P, Meyers DJ, $\mathrm{Xu}$ J. Prevalence of interstitial cystitis in first-degree relatives of patients with interstitial cystitis. Urol. 2004;63(1):17-21.

26. Clemens JQ, Meenan RT, Rosetti MC, Gao SY, Calhoun EA. Prevalence and incidence of interstitial cystitis in a managed care population. $\mathbf{J}$ Urol. 2005;173(1):98-102.

27. Shie JH, Liu HT, Kuo HC. Increased cell apoptosis of urothelium mediated by inflammation in interstitial cystitis/painful bladder syndrome. Urol. 2012;79(2):484.e7-484.e13.

28. Gu D, Huang J, Yin Y, Shan Z, Zheng S, Wu P. Long-term ketamine abuse induces cystitis in rats by impairing the bladder epithelial barrier. Mol Biol Rep. 2014;41(11):7313-22.

29. Nickel JC, Tripp DA, Pontari M. Childhood sexual trauma in women with interstitial cystitis/bladder pain syndrome: a case control study. Can Urol Assoc J. $2011 ; 5(6): 410-5$.

30. Lorenzo Gómez MF, Gómez Castro S. Physiopathologic relationship between interstitial cystitis and rheumatic, autoimmune, and chronic inflammatory diseases. Arch Esp Urol. 2004;57(1):25-34.

31. Eric S, Rovner MD. Interstitial cystitis: etiology. MedScape Reference. Available at http://emedicine.medscape.com/article/2055505overview.

32. Hanno PM, Burks DA, Clemens JQ. AUA guideline for the diagnosis and treatment of interstitial cystitis/bladder pain syndrome. J Urol. 2011;185(6):2162-70.

33. Hanno PM, Burks DA, Clemens JQ. Diagnosis and treatment of interstitial cystitis/bladder pain syndrome. American Urological Association. Available at https:// www.auanet.org / education / guidelines/ic-bladder-pain-syndrome.cfm. Accessed on 21 September 2015.

34. Parker KS, Crowley JR, Stephens-Shields AJ, van Bokhoven A, Lucia MS, Lai HH, et al. Urinary Metabolomics Identifies a Molecular Correlate of Interstitial Cystitis/Bladder Pain Syndrome in a Multidisciplinary Approach to the Study of Chronic Pelvic Pain (MAPP) Research Network Cohort. E Bio Medicine. 2016;7:167-74.

35. Warren JW, Meyer WA, Greenberg P. Using the International Continence Society's definition of painful bladder syndrome. Urol. 2006;67:1138.

36. Fitzgerald MP, Koch D, Senka J. Visceral and cutaneous sensory testing in patients with painful bladder syndrome. Neurourol Urodyn. 2005;24:627.

37. Sirinian E, Payne CK. Correlation of symptoms between 2 instruments among interstitial cystitis patients. Urol. 2001;57:124.

38. Warren JW, Brown J, Tracy JK. Evidence-based criteria for pain of interstitial cystitis/painful bladder syndrome in women. Urol. 2008;71:444.

39. Tincello DG, Walker AC. Interstitial cystitis in the UK: results of a questionnaire survey of members of the Interstitial Cystitis Support Group. Eur J Obstet Gynecol Reprod Biol. 2005;118:91.

40. Diggs C, Meyer WA, Langenberg P. Assessing urgency in interstitial cystitis/ painful bladder syndrome. Urol. 2007;69:210.

41. Greenberg P, Tracy JK, Meyer WA. Short interval between symptom onset and medical care as an indication of rapid onset of interstitial cystitis/painful bladder syndrome. BJU Int. 2007;100:599.

42. Dyer AJ, Twiss CO. Painful bladder syndrome: an update and review of current management strategies. Curr Urol Rep. 2014;15(2):384.

43. Bogart LM, Berry SH, Clemens JQ. Symptoms of interstitial cystitis, painful bladder syndrome and similar diseases in women: a systematic review. The J Urol. 2007;177(2):450-6.

44. Gupta P, Gaines N, Sirls LT, Peters KM. A multidisciplinary approach to the evaluation and management of interstitial cystitis/bladder pain syndrome: an ideal model of care. Transl Androl Urol. 2015;4(6):611-9.

45. Hanno P, Lin A, Nordling J. Bladder pain syndrome committee of the International consultation on incontinence. Neurourol Urodyn. 2010;29(1):191-8.

46. Tyagi P, Kashyap MP, Kawamorita N, Yoshizawa T, Chancellor M, Yoshimura N. Intravesical liposome and antisense treatment for detrusor overactivity and 
interstitial cystitis/painful bladder syndrome. ISRN Pharmacol. 2014;(601653):1-12.

47. Cho YS. Interstitial Cystitis/Bladder Pain Syndrome: A Urologic Mystery. Int Neurourol J. 2016;20(1):3-4.

48. Colaco M, Evans R. Current guidelines in the management of interstitial cystitis. Transl Androl Urol. 2015;4(6):677-83.

49. Cvach K, Rosamilia A. Review of intravesical therapies for bladder pain syndrome/ interstitial. Transl Androl Urol. 2015;4(6):629-37

50. Oh HM, Chung ME. Botulinum toxin for neuropathic pain: a review of the literature. Toxins (Basel). 2015;7:3127-54.

51. Chiu B, Tai HC, Chung SD, Lori A. Birder 3,4 Botulinum Toxin A for Bladder Pain Syndrome/Interstitial Cystitis. Toxins. 2016;8:201.

52. Barr S. Diagnosis and management of interstitial cystitis. Obstet Gynecol Clin North Am. 2014;41:397-407.

53. Harris N. Treatment of bladder urothelium injury. Urologia. 2015 Oct;82 Suppl 3:S6-9.

54. Colaco M, Evans R. Current guidelines in the management of interstitial cystitis. Transl Androl Urol. 2015;4(6):677-83.

55. Oduah E, Linhardt RJ, Sharfstein ST. Heparin: past, present, and future. Pharma. 2016;9(3):38.

56. Nickel JC, Moldwin R, Lee S. Intravesical alkalinized lidocaine (PSD597) offers sustained relief from symptoms of interstitial cystitis and painful bladder syndrome. BJUInt. 2009;103:910-8.

57. Chiang G, Patra P, Letourneau R. Pentosanpolysulfate inhibits mast cell histamine secretion and intracellular calcium ion levels: an alternative explanation of its beneficial effect in interstitial cystitis. J Urol. 2000;164:2119-25.

58. Daha LK, Riedl CR, Lazar D. Do cystometric findings predict the results of intravesical hyaluronic acid in women with interstitial cystitis? Eur Urol 2005;47:393-7.

59. Jhang JF, Kuo HC. Botulinum toxin A and lower urinary tract dysfunction: pathophysiology and mechanismsof action. Toxins. 2016;8:120.

60. Payne CK, Mosbaugh PG, Forrest JB. Intravesical resiniferatoxin for the treatment of interstitial cystitis: a randomized, double-blind, placebo controlled trial. J Urol. 2005;173:1590-4.

61. Propert KJ, Mayer R, Nickel JC. Followup of patients with interstitial cystitis responsive to treatment with intravesical bacillus Calmette-Guerin or placebo. J Urol. 2008;179:552-5.

62. Chuang YC, Lee WC, Lee WC. Intravesical liposome versus oral pentosan polysulfate for interstitial cystitis/ painful bladder syndrome. J Urol. 2009; 182:1393-400.

63. Payne RA, O'Connor RC, Kressin M. Endoscopic ablation of Hunner's lesions in interstitial cystitis patients. Can Urol Assoc J. 2009;3:473-7.

64. Hillelsohn JH, Rais-Bahrami S, Friedlander JI. Fulguration for Hunner ulcers: long-term clinical outcomes. J Urol. 2012;188:2238-41.

65. Hsieh $\mathrm{CH}$, Chang WC, Huang MC, Su TH, Li YT, Chiang HS. Treatment of interstitial cystitis in women. Taiwan J Obstet Gynecol. 2012;51(4):52632.

66. Forrest JB. Economics of interstitial cystitis in clinical practice. Rev Urol. 2002;4 Suppl 1:S44-8.

67. Büssing A, Ostermann T, Lüdtke R. Effects of yoga interventions on pain and pain-associated disability: a meta-analysis. J Pain. 2012;13:1-9.

68. Hanno PM, Burks DA, Clemens JQ. AUA guideline for the diagnosis and treatment of interstitial cystitis/ bladder pain syndrome. J Urol. 2011;185:2162-70.

69. Gamé X, Rischmann P, Arnal JF. Nitric oxide pathway and female lower urinary tract. Physiological and pathophysiological role. Prog Urol. 2013;23:926-35.

70. Pang R, Ali A. The Chinese approach to complementary and alternative medicine treatment for interstitial cystitis/bladder pain syndrome Transl Androl Urol. 2015;4(6):653-61.

71. Tempest H, Reynard J, Bryant RJ. Acupuncture in urological practice-a survey of urologists in England. Complement Ther Med. 2011;19:27-31.

Cite this article as: Shebl SE, Dawood AS, Dawood AS. Bladder pain syndrome from urogynecological point of view: a narrative. Int J Reprod Contracept Obstet Gynecol 2017;6:1694-702. 